\title{
Practical clinical tool to monitor dementia symptoms: the HABC-Monitor
}

\author{
This article was published in the following Dove Press journal: \\ Clinical Interventions in Aging \\ 13 June 2012 \\ Number of times this article has been viewed
}

\author{
Patrick O Monahan' \\ Malaz A Boustani ${ }^{1-3,6}$ \\ Catherine Alder $2,3,6$ \\ James E Galvin ${ }^{7}$ \\ Anthony J Perkins ${ }^{2,3}$ \\ Patrick Healey ${ }^{4}$ \\ Azita Chehresa ${ }^{5}$ \\ Polly Shepard ${ }^{8}$ \\ Corby Bubp ${ }^{8}$ \\ Amie Frame ${ }^{2,3}$ \\ Chris Callahan ${ }^{1-3,6}$ \\ 'Indiana University School of \\ Medicine, ${ }^{2}$ Indiana University Center \\ for Aging Research, ${ }^{3}$ Regenstrief \\ Institute Inc, ${ }^{4}$ St Vincent's Health \\ Network, ${ }^{5}$ Community Health \\ Network, ${ }^{6} \mathrm{~W}$ ishard Health Services, \\ Eskenazi Health, Indianapolis IN, \\ ${ }^{7}$ Alzheimer Disease Center, \\ Departments of Neurology and \\ Psychiatry, New York University \\ School of Medicine, New York, NY, \\ ${ }^{8}$ Memory Clinic of Indianapolis, \\ Indianapolis, IN, USA
}

Correspondence: Malaz A Boustani Regenstrief Institute Inc, 4 I 0 West I0th Street, Suite 2000, Indianapolis, IN 46202-30I 2, USA

$\mathrm{Tel}+\mathrm{I} 3174235633$

Fax +I 3174235695

Email mboustan@iupui.edu
Background: Dementia care providers need a clinical assessment tool similar to the blood pressure cuff (sphygmomanometer) used by clinicians and patients for managing hypertension. A "blood pressure cuff" for dementia would be an inexpensive, simple, user-friendly, easily standardized, sensitive to change, and widely available multidomain instrument for providers and informal caregivers to measure severity of dementia symptoms. The purpose of this study was to assess the reliability and validity of the Healthy Aging Brain Care Monitor (HABC-Monitor) for measuring and monitoring the severity of dementia symptoms through caregiver reports.

Methods: The first prototype of the HABC-Monitor was developed in collaboration with the Indianapolis Discovery Network for Dementia, which includes 200 members representing 20 disciplines from 20 local organizations, and an expert panel of 22 experts in dementia care and research. The HABC-Monitor has three patient symptom domains (cognitive, functional, behavioral/psychological) and a caregiver quality of life domain. Patients $(n=171)$ and their informal caregivers $(n=171)$ were consecutively approached and consented during, or by phone shortly following, a patient's routine visit to their memory care provider.

Results: The HABC-Monitor demonstrated good internal consistency (0.73-0.92); construct validity indicated by correlations with the caregiver-reported Neuropsychiatric Inventory (NPI) total score and NPI caregiver distress score; sensitivity to three-month change compared with NPI "reliable change" groups; and known-groups validity, indicated by significant separation of Mini-Mental Status Examination severity groups and clinical diagnostic groups. Although not designed as a screening study, there was evidence for good operating characteristics, according to area under the receiver-operator curve with respect to gold standard clinical diagnoses, relative to Mini-Mental Status Examination or NPI.

Conclusion: The HABC-Monitor demonstrates good reliability and validity as a clinically practical multidimensional tool for monitoring symptoms of dementia through the informal caregiver.

Keywords: dementia, symptoms, monitor, validation, cognitive impairment, memory care

\section{Introduction}

Dementia is a complex brain syndrome with a spectrum of cognitive, functional, behavioral, and psychological symptoms that reduces quality of life for both patients and their informal caregivers. ${ }^{1,2}$ Behavioral and psychological symptoms related to dementia are among the most difficult symptoms of the syndrome to manage. ${ }^{3}$ Two recent randomized controlled trials ${ }^{4,5}$ demonstrated the effectiveness of using a collaborative care model to reduce both behavioral and psychological symptoms related to dementia in patients and the stress for their informal caregivers. ${ }^{4-7}$ However, the success of this 
model required continuous monitoring of both the dementia symptoms and the effectiveness of the individualized care protocols utilized in managing these symptoms..$^{4-7}$ Enhancing dementia care by implementing a collaborative dementia care model demands the development of a practical, accurate, sensitive to change, and multidomain clinical tool effective both in identifying the biopsychosocial needs of patients with dementia and in detecting any changes occurring in response to the care plan implemented by clinicians. Ideally, dementia care providers need a clinical tool similar to the blood pressure cuff (sphygmomanometer) used by clinicians, patients, and their family members for the recognition and management of hypertension. A "blood pressure cuff" for dementia would be an inexpensive, simple, user-friendly, easily standardized, and widely available instrument for both providers and informal caregivers to measure and track dementia symptoms.

There are many tools available today to gauge the presence and severity of dementia symptoms through caregiver observation and report. However, most are best suited for research studies and not clinical practice because they are lengthy and/or cover only particular domains. For example, the Clinical Dementia Rating ${ }^{8}$ is one of the gold standards for assessing severity of dementia but is quite extensive and does not include the behavioral and psychological symptoms related to dementia. Conversely, other tools exist for measuring behavioral and psychological symptoms related to dementia, including the Neuropsychiatric Inventory (NPI), which has become a widely used instrument for this purpose. ${ }^{9}$ However, the time taken to complete the NPI is impractical for most clinic settings and the NPI does not cover functional or cognitive domains. Many additional tools are available to screen specifically for cognitive impairment. ${ }^{2}$ Among instruments that contain more than one domain and intended to have a short administration time, the Dementia Severity Rating Scale ${ }^{10}$ assesses the cognitive and functional domains but not the behavioral domain and is lengthier than the HABC-Monitor because the Dementia Severity Rating Scale requires several pages as each of the 12 items has a different set of detailed response options. The 47-item Dementia Severity Scale ${ }^{11}$ covers the cognitive, functional, and behavioral domains, but does not contain mood (depression or anxiety) symptoms.

Over the last 3 years, we have constructed a prototype of a "blood pressure cuff" for dementia. We have assessed its face and content validity based on the input of real-world users and an international panel of dementia care experts. On the advice of our expert panel, we sought to avoid the potential negative connotation of the word "dementia" by giving our instrument a name that uses safe, positive, simple, and familiar language, ie, the Healthy Aging Brain Care Monitor (HABC-Monitor or HABC-M). This study was undertaken to determine whether the HABC-Monitor exhibits the psychometric properties of reliability and validity necessary for broad clinical use.

\section{Methods}

\section{Instrument development}

The first prototype of the HABC-Monitor was developed in collaboration with the Indianapolis Discovery Network for Dementia, which is a local, diverse, and sustainable network dedicated to enhancing the quality of life and care of individuals with dementia. The network includes over 200 members representing 20 disciplines from 20 local organizations, including the four largest health care systems in Indianapolis. Building the HABC-Monitor was accomplished through a series of steps.

With the support of the Indianapolis Discovery Network for Dementia and an unrestricted research grant from Forest Pharmaceuticals, we constructed an interdisciplinary expert panel team of 22 representatives from three disciplines (clinical care, clinical research, and psychometrics) involved in dementia care and research. The clinical team included four primary care physicians, three dementia physicians (a neurologist and two geriatricians), two nurse providers, a pharmacist, and a social worker. The clinical research team included a dementia health services researcher, a dementia epidemiologist, a dementia care coordinator, three neuropsychologists, and two clinical trialists. The psychometric team included three psychometricians. In September 2008, we coordinated and led an offsite two-day retreat to assess the face and content validity as well as feasibility and usability of the instrument. Following the retreat, we contacted all participants by email seeking their subsequent feedback. The expert panel agreed that the target instrument should include items covering four clusters including dementia symptoms (cognitive, functional, and behavioral/psychological) and caregiver quality of life to accomplish the primary goals of measuring severity of symptoms and assessing response to therapy. In a systematic evidence review of the literature, the team identified questions from existing scales to tap the four clusters and created a common item response set that is simple and sensitive to variation over time and treatment. The HABC-Monitor items were selected by consensus amongst the 22 members of our expert panel. The panel elected to use 23 items from legacy instruments consisting of 17 items that best capture the cognitive, behavioral, and 
psychological symptoms of dementia (ie, items 1-7 and 18-27, see Appendix) including all eight items from the AD8, ${ }^{12,13}$ four items from the PHQ- $9,{ }^{14}$ ten items from the NPI-Q, ${ }^{15}$ and six instrumental and basic activities of daily living items from the 12-item functional survey used in the Assessing Care of Vulnerable Elders study (ie, items 8-13). ${ }^{16}$ Four of the selected items from the AD8, PHQ-9, and NPI-Q overlap. Namely, the second item of AD8 and the seventh item of the NPI-Q are also measured by the first item of the PHQ-9, and all four selected PHQ-9 items are contained in the NPI-Q. The rationale for including these 23 items was that these items were derived from well validated and clinically relevant instruments that capture the cognitive, functional, behavioral, and mood symptoms of patients with dementia and that these items could be translated into items easily reported by informal caregivers. The rationale for the panel to develop eight new items was to capture additional functional symptoms (eg, safety and falling, items 14-17) related to dementia and items that capture the quality of life of the informal caregiver (items 28-31) that is impacted by dementia-related disability. Furthermore, the experts structured the item response options using four ordinal categories to allow measurement of variation over time and to reach consistency in the response set across questions.

Although the panel recognized the importance and value of using clinician observers or performance tests as a possible source of data relevant to dementia care, the panel decided to focus on practical and feasible sources of data relying on the perceptions and observations of the patients' informal caregivers. In addition, the team discussed the various vehicles that could be used to capture data from caregivers and opted to develop a flexible template capable of accommodating paper, telephone, or web-based data entry. The expert team recognized that the relative benefit of each of the four domains of the HABC-Monitor will vary based on the clinical objective. As an example, cognitive and functional domains may facilitate diagnosis, whereas caregiver stress and behavioral and psychological symptoms may contribute to the measurement of response to therapy. All procedures were approved by the institutional review board of the Indiana University-Purdue University campus in Indianapolis.

\section{Clinical setting and population}

Our study was conducted at the Healthy Aging Brain Center (HABC), a memory care practice located within Wishard Health Services, a safety net health care system primarily serving an urban, racially and ethnically mixed population of vulnerable adults. ${ }^{6}$ This memory care practice is one of five memory care practices affiliated with the Indianapolis Discovery Network for Dementia. The HABC staff includes four memory care practitioners, four care coordinators (two social workers and two registered nurses), two medical assistants, and one technician skilled in neuropsychological testing. The care coordinator conducts a previsit structured telephone interview with the informal caregiver to collect the necessary information related to the patient's cognitive, functional, behavioral, and psychological symptoms, as well as caregiver burden. The HABC technician administers the Consortium to Establish a Registry for Alzheimer's Disease neuropsychological battery to every new patient seen in the HABC. The memory care physician performs a structured physical and neurological examination, and orders any necessary laboratory and brain imaging tests. Data collected through these processes are used collectively by the HABC team to make a diagnosis of dementia, mild cognitive impairment, or normal cognitive status. ${ }^{6}$ Furthermore, the care coordinators provide ongoing telephone support and care management for both patients and caregivers seen in the $\mathrm{HABC}$. During each clinic visit and telephone contact, the care coordinator assesses for cognitive, functional, behavioral, and psychological symptoms in the patient and for caregiver stress. In addition, care coordinators are responsible for contacting the patient's primary care physician to facilitate medical comanagement and for coordinating with local resources, such as the local Alzheimer's Association. The mean age of patients seen in our HABC is 75 years, and $72 \%$ are female, $39 \%$ are underrepresented minorities, and $80 \%$ are Medicare beneficiaries. Using Diagnostic and Statistical Manual of Mental Disorders, Fourth Edition, Text Revision criteria, $32 \%$ of the patients seen in the HABC have probable or possible Alzheimer disease, $10 \%$ have vascular dementia, $33 \%$ have mild cognitive impairment, and $25 \%$ are normal or have other cognitive or mood disorders. ${ }^{6}$

\section{Subject recruitment and testing}

During a patient's routine HABC visit, a research assistant approached the patient and the patient's caregiver, or contacted them by phone shortly thereafter, to obtain informed consent for participation in this study. All of the HABC providers agreed to allow their patients and caregivers to be approached. Each subject (caregiver) was asked to complete the HABC-Monitor and the Neuropsychiatric Inventory. Each patient was asked to complete the PHQ-9. The test batteries were repeated 3 months later. The two batteries were administered by a research assistant and completed either face-to-face or via telephone. 


\section{Assessment questionnaires HABC-Monitor}

The current HABC-Monitor includes 31 items covering four clinically relevant domains of dementia, ie, cognitive, functional, behavioral, and psychological symptoms, and caregiver quality of life. For brevity and practical use in the clinical setting, each item on the four scales was designed to have the same item response options consisting of four categories that use the frequency of the target problem in the past 2 weeks. The appendix outlines all of the items of the HABC-Monitor instrument. A public website hosts our instrument (http://www.wishard.edu/our-services/senior-care/ healthy-aging-brain-center/cgm). The HABC-Monitor took approximately 6 minutes to complete.

\section{Neuropsychiatric inventory}

The NPI is based on a structured interview administered to an informal caregiver and has been adopted by the Alzheimer's Disease Cooperative Studies Group to obtain information on the presence of psychopathology in behavioral areas including delusions, apathy, hallucinations, disinhibition, agitation, depression, aberrant motor behavior, anxiety, night-time behavior, and euphoria. ${ }^{9}$ For each of 12 symptoms, if the caregiver reports the presence of psychopathology, a frequency and severity score are multiplied to yield a possible item score range of $0-12$, and a possible total score range of $0-144$. The NPI can be used to assess changes in the patient's behavior over the past month. The NPI also assesses the level of caregiver distress attributable to each of the 12 patient behaviors, with a possible total caregiver distress score range of $0-60$. The administration time is about 20 minutes. The NPI has excellent reliability and validity. ${ }^{9}$

\section{Other data collection}

The research assistants reviewed the memory care practice medical records of each participating patient to record the clinical diagnosis made by the HABC team, ie, dementia, mild cognitive impairment, or normal. The Mini-Mental Status Examination (MMSE) ${ }^{17}$ score was collected from the Consortium to Establish a Registry for Alzheimer's Disease battery during the initial visit to the HABC. Patient and caregiver age, gender, race and ethnicity, and the highest level of formal education completed by the caregiver, were also collected.

\section{Scaling procedure}

Each HABC-Monitor scale score was computed by summing all items in the scale. A higher score represented a worse score for all HABC-Monitor scales and total score. When computing scale scores, a person-specific and scale-specific mean of nonmissing items was substituted for missing items if $50 \%$ or fewer of the items on the scale were missing.

\section{Statistical analysis}

Confirmatory factor analysis was performed using MPLUS software version 5.21 (Muthen and Muthen, Los Angeles, CA).${ }^{18}$ All other analyses were performed with SAS version 9.3 (SAS Institute Inc, Cary, NC).

\section{Data quality and descriptive analyses}

Data completeness was assessed by calculating missing data rates for each item. To assess item variability, the item frequency distributions, range, and standard deviations were calculated. Item and scale scores were examined for floor and ceiling effects (ie, clustering of participants at the best and worst possible perceptions, respectively). To determine the range of caregiver perceptions measured by the HABC-Monitor scales, the observed score range and measures of central tendency and variability were computed.

\section{Psychometric analyses}

Confirmatory factor analysis was performed with MPLUS to confirm the hypothesized factor structure of the HABCMonitor item responses. Criteria of good model fit were the following: comparative fit index $>0.95,{ }^{19}$ root mean square error of approximation $<0.06,{ }^{19}$ and weighted root mean square residual $<1.00 .{ }^{20}$ Modification indices were used to determine whether fit of the confirmatory factor analysis model could be improved by adding any paths or cross-loadings. The items were appropriately modeled as ordinal categorical (not continuous) items. The strength of association between individual items and the hypothesized factors were assessed with item standardized factor loadings. All four factors were hypothesized to be correlated with each other. Internal-consistency reliability was estimated with coefficient alpha. ${ }^{21}$ Reliability of 0.70 or greater was considered satisfactory for group comparison purposes. ${ }^{22}$ Sensitivity of the HABC-Monitor scores to change was assessed. ${ }^{23,24}$ Specifically, because the NPI and HABC-Monitor were both administered to caregivers at baseline and at 3 months, and because the NPI total score is considered a gold standard for the purpose of assessing psychopathology in behavioral areas relevant to dementia," "reliable change" groups of NPI total score (decline, stable, improve) were computed by determining for each patient whether the caregivers' NPI 
total score declined or improved by more than 1.0 standard error of measurement (SEM) from baseline to follow-up at 3 months. One SEM was defined as the standard deviation of change scores for the NPI total, multiplied by the square root of [1.0 minus reliability], where reliability was specified as 0.79 , ie, the published test-retest reliability coefficient of the NPI total score. ${ }^{25}$ For each reliable change group, the effect size for the sensitivity to change for the HABC-Monitor total and subscale scores were computed as the standardized response mean: M2 - M1/SDdiff $($ SDdiff = standard deviation of score changes). The three NPI reliable change groups were statistically compared on the HABC-Monitor change scores using analysis of variance, with pairwise Tukey-Kramer post hoc tests which controlled the familywise Type I error rate at 0.05 .

Known-groups validity was assessed by using analysis of variance to compare HABC-Monitor scores between patient groups formed by MMSE scores (0-9, 10-17, 18-23, 24+) and gold standard clinical diagnoses (normal, cognitive impairment, dementia). A significant omnibus $\mathrm{F}$ test was followed by pairwise Tukey-Kramer post hoc tests with 0.05 familywise Type I error.

This study was not designed to be a screening study. For example, in a screening study, we would have purposively enrolled a larger number of patients in the "normal" diagnostic category. Nevertheless, we provided a brief description of preliminary data for the operating characteristics of the HABC-Monitor scales, namely, the area under the receiver-operator curve (AUROC) with respect to gold standard clinical diagnostic groups (normal, mild cognitive impairment, dementia).

\section{Results}

\section{Demographics}

Of 266 patient and caregiver dyads approached, 171 signed the informed consent and Health Insurance Portability and Accountability Act documents and completed baseline assessments. Patient and caregiver characteristics are shown in Table 1. Patients on average were 76 years of age; a majority were female (63\%) and a substantial minority were African American (38\%). Their informal caregivers were on average 58 years of age, mostly female (76\%), and $58 \%$ had more than a high school education (see Table 1).

\section{Data quality}

Table 2 demonstrates that all items of the HABC-Monitor tool exhibited the full range of response categories across the four item response options. In general, the item responses
Table I Participant characteristics

\begin{tabular}{|c|c|}
\hline \multicolumn{2}{|c|}{ Patient characteristics } \\
\hline Age, mean (SD) & $75.7(10.3)$ \\
\hline \multicolumn{2}{|l|}{ Gender } \\
\hline Female & $63 \%$ \\
\hline Male & $37 \%$ \\
\hline \multicolumn{2}{|l|}{ Race } \\
\hline White & $61 \%$ \\
\hline African-American & $38 \%$ \\
\hline Other & $1 \%$ \\
\hline \multicolumn{2}{|l|}{ Ethnicity } \\
\hline Hispanic & $1 \%$ \\
\hline Not Hispanic & $99 \%$ \\
\hline \multicolumn{2}{|c|}{ Caregiver characteristics } \\
\hline \multicolumn{2}{|c|}{ How well caregiver knows patient } \\
\hline Very well & $90 \%$ \\
\hline Well & $10 \%$ \\
\hline \multicolumn{2}{|c|}{ Relationship of caregiver to patient } \\
\hline Spouse or partner & $34 \%$ \\
\hline Child & $52 \%$ \\
\hline Grandchild & $4 \%$ \\
\hline Parent & $2 \%$ \\
\hline Sibling & $6 \%$ \\
\hline Other & $2 \%$ \\
\hline Age, mean (SD) & $57.7(14.1)$ \\
\hline \multicolumn{2}{|l|}{ Gender } \\
\hline Female & $76 \%$ \\
\hline Male & $24 \%$ \\
\hline \multicolumn{2}{|l|}{ Race } \\
\hline White & $61 \%$ \\
\hline African-American & $38 \%$ \\
\hline Other & $1 \%$ \\
\hline \multicolumn{2}{|l|}{ Ethnicity } \\
\hline Hispanic & $1 \%$ \\
\hline Not Hispanic & $99 \%$ \\
\hline \multicolumn{2}{|l|}{ Education } \\
\hline $0-1 \mid$ Years & $14 \%$ \\
\hline 12 Years & $28 \%$ \\
\hline $13+$ Years & $58 \%$ \\
\hline
\end{tabular}

were more heavily distributed among the 0 and 1 scores than the 2 and 3 scores, as indicated by item means that ranged from 0.3 to 1.7 . Missing item rates were very low and ranged from $0 \%(n=171)$ to $1.8 \%(n=168)$, as shown in Table 2 . The item-level floor effects ranged from $16 \%$ to $82 \%$ with a median of $68 \%$. The item-level ceiling effects ranged from $2 \%$ to $35 \%$ with a median of $12 \%$. The standard deviation was similar for all items, ranging from 0.7 to 1.2. Thus, data quality of the HABC-Monitor was satisfactory.

\section{Confirmatory factor analysis}

We tested two alternative or competing models that were specified a priori before analyses and differed only in whether the anhedonia item (item 18, "less interest or pleasure in doing things, hobbies or activities") was hypothesized to 
Table 2 Item distributions, missing rates, confirmatory factor analysis (CFA), and item-total correlations

\begin{tabular}{|c|c|c|c|c|c|c|c|c|c|}
\hline \multirow[t]{3}{*}{ HABC-M domains and items } & \multicolumn{6}{|c|}{ Item distribution and missing rates } & \multirow[t]{3}{*}{$\%$ miss } & \multirow[t]{3}{*}{ CFA } & \multirow{3}{*}{$\begin{array}{l}\text { Item-total } \\
\text { Pearson } r\end{array}$} \\
\hline & \multicolumn{2}{|l|}{ Item } & \multicolumn{4}{|c|}{ Response counts } & & & \\
\hline & Mean & SD & 0 & I & 2 & 3 & & & \\
\hline \multicolumn{7}{|l|}{ Cognitive domain (factor I) } & & \multicolumn{2}{|c|}{ Factor I } \\
\hline I. Judgment or decision-making & 1.3 & 1.2 & 52 & 54 & 22 & 43 & 0.0 & 0.87 & 0.72 \\
\hline $\begin{array}{l}\text { 2. Repeating the same things over and over such } \\
\text { as questions or stories }\end{array}$ & I.I & I.I & 72 & 41 & 29 & 29 & 0.0 & 0.78 & 0.65 \\
\hline 3. Forgetting the correct month or year & 1.5 & 1.2 & 54 & 39 & 22 & 56 & 0.0 & 0.65 & 0.59 \\
\hline $\begin{array}{l}\text { 4. Handling complicated financial affairs such as } \\
\text { balancing checkbook, income taxes and paying bills }\end{array}$ & 0.6 & 1.0 & 118 & 24 & 12 & 17 & 0.0 & 0.71 & 0.52 \\
\hline 5. Remembering appointments & 1.0 & 1.2 & 87 & 36 & 15 & 33 & 0.0 & 0.83 & 0.70 \\
\hline 6. Thinking or memory & 0.8 & 1.2 & 109 & 17 & 10 & 35 & 0.0 & 0.81 & 0.68 \\
\hline \multicolumn{8}{|l|}{ Functional domain (factor 2) } & \multicolumn{2}{|c|}{ Factor 2} \\
\hline 7. Learning to use a tool, appliance, or gadget & I.I & 1.2 & 79 & 34 & 18 & 40 & 0.0 & 0.69 & 0.55 \\
\hline 8. Planning, preparing, or serving meals & 1.7 & I.I & 28 & 53 & 30 & 59 & 0.6 & 0.78 & 0.63 \\
\hline 9. Taking medications in the right dose at the right time & 0.6 & 1.0 & 123 & 18 & 8 & 21 & 0.6 & 0.67 & 0.51 \\
\hline 10. Walking or physical ambulation & 0.7 & I.I & 115 & 23 & 5 & 27 & 0.6 & 0.32 & 0.24 \\
\hline II. Bathing & 0.8 & I.I & 100 & 30 & 15 & 26 & 0.0 & 0.82 & 0.61 \\
\hline 12. Shopping for personal items like groceries & 0.4 & 1.0 & 138 & 8 & 9 & 16 & 0.0 & 0.92 & 0.74 \\
\hline 13. Housework or household chores & 0.5 & 1.0 & 127 & 20 & 2 & 22 & 0.0 & 0.88 & 0.76 \\
\hline 14. Leaving her/him alone & 0.5 & 1.0 & 128 & 12 & 14 & 17 & 0.0 & 0.83 & 0.59 \\
\hline 15. Her/his safety & 0.4 & 0.9 & 133 & 16 & 8 & 14 & 0.0 & 0.93 & 0.69 \\
\hline 16. Her/his quality of life & 0.4 & 0.9 & 134 & 18 & 6 & 12 & 0.6 & 0.67 & 0.52 \\
\hline 17. Falling or tripping & 0.7 & 1.0 & 102 & 32 & 20 & 16 & 0.6 & 0.56 & $0.4 \mathrm{I}$ \\
\hline \multicolumn{8}{|l|}{ Behavioral and psychological domain (factor 3 ) } & \multicolumn{2}{|c|}{ Factor 3} \\
\hline $\begin{array}{l}\text { 18. Less interest or pleasure in doing things, hobbies } \\
\text { or activities }\end{array}$ & 0.3 & 0.7 & 132 & 29 & 6 & 4 & 0.0 & 0.68 & 0.49 \\
\hline 19. Feeling down, depressed, or hopeless & 1.0 & 1.1 & 78 & 49 & 18 & 26 & 0.0 & 0.60 & 0.55 \\
\hline \multicolumn{10}{|l|}{$\begin{array}{l}\text { 20. Being stubborn, agitated, aggressive or resistive } \\
\text { to help from others }\end{array}$} \\
\hline 21. Feeling anxious, nervous, tense, fearful or panic & 0.8 & $\mathrm{I} . \mathrm{I}$ & 99 & 36 & 14 & 22 & 0.0 & 0.76 & 0.60 \\
\hline $\begin{array}{l}\text { 22. Believing others are stealing from them or planning } \\
\text { to harm them }\end{array}$ & 0.3 & 0.8 & 140 & 15 & 6 & 10 & 0.0 & 0.67 & 0.53 \\
\hline $\begin{array}{l}\text { 23. Hearing voices, seeing things or talking to people } \\
\text { who are not there }\end{array}$ & 0.3 & 0.7 & 138 & 22 & 3 & 8 & 0.0 & 0.62 & 0.39 \\
\hline 24. Poor appetite or overeating & 0.8 & 1.2 & 102 & 30 & 9 & 30 & 0.0 & 0.64 & 0.43 \\
\hline 25. Falling asleep, staying asleep, or sleeping too much & 1.0 & I.I & 80 & 42 & 18 & 30 & 0.6 & 0.29 & 0.33 \\
\hline $\begin{array}{l}\text { 26. Acting impulsively, without thinking through } \\
\text { the consequences of her/his actions }\end{array}$ & 0.5 & 0.9 & 123 & 25 & 10 & 13 & 0.0 & 0.84 & 0.56 \\
\hline 27. Wandering, pacing, or doing things repeatedly & 0.5 & 1.0 & $|3|$ & 16 & 6 & 18 & 0.0 & 0.82 & 0.55 \\
\hline Caregiver quality of life domain (factor 4) & & & & & & & & Factor & \\
\hline 28. Your quality of life & 0.6 & 0.9 & III & 34 & 11 & 12 & 1.8 & 0.95 & 0.50 \\
\hline 29. Your financial future & 0.5 & 0.9 & 127 & 21 & 8 & 13 & 1.2 & 0.46 & 0.48 \\
\hline 30. Your mental health & 0.3 & 0.7 & 135 & 24 & 5 & 5 & 1.2 & 0.86 & 0.59 \\
\hline 31. Your physical health & 0.5 & 0.9 & 121 & 25 & 9 & 14 & 1.2 & 0.60 & 0.53 \\
\hline Fit statistics from confirmatory factor analysis (CF & & & & & & RMSEA & & CFI & WRMR \\
\hline Model I (a priori hyothesized model, anhedonia item in fac & or I not & ctor 3) & & & & 0.062 & & 0.923 & 1.082 \\
\hline Model 2 (a priori hyothesized model, anhedonia item in fac & or 3 not & ctor I) & & & & 0.062 & & 0.923 & 1.078 \\
\hline Model 3 (final revised model, same as model 2 except learnir & gadget it & $n$ in fact & or $2 n$ & facto & & 0.059 & & 0.929 & 1.055 \\
\hline
\end{tabular}

Notes: All items had a four-category response scale: $0=$ None at all (0-I day), I = Several Days (2-6 days), $2=$ More than half the days $(7-I I$ days), $3=$ Almost daily (12-14 days). \% miss = \% of participants missing the item.

Abbreviations: HABC-M, Healthy Aging Brain Care Monitor; RMSEA, root mean square error of approximation; WRMR, weighted root mean square residual.

load on the cognitive factor (factor 1) or the behavioral and psychological factor (factor 3 ). The other two factors in the confirmatory factor analysis model were the functional factor (factor 2) and the caregiver quality of life factor (factor 4). The fit statistics were very similar for the two competing models and for the final model; therefore, decisions about the final model were made on the basis of the magnitude and significance of item loadings and conceptual relevance. The loading for the anhedonia item had similar magnitude $(0.65$ and 0.68 ), and was significant in both models; however, 
when this item was allowed to cross-load on both factor 1 and factor 3 , the anhedonia item was not significant on the cognitive factor (factor 1 , loading $=0.25, P=0.068$ ) but was significant on the behavioral and psychological factor (factor 3, loading $=0.43, P=0.004$ ). Therefore, due to this finding and the fact that anhedonia is more commonly classified as a mood symptom, we decided to place the item in the behavioral and psychological factor. Only one of the modification indices from the two alternative models displayed conceptual relevance. This modification index suggested that the following path could be significant if added to the model: functional factor to item 7, "learning to use a tool, appliance, or gadget". This was investigated by allowing the learning gadget item to cross-load on both the cognitive and functional factors. The learning gadget item was found to be not significant on the cognitive factor (loading $=-0.16, P=0.43$ ) but highly significant on the functional factor (loading $=0.84, P<0.0001$ ). Therefore, due to this and conceptual relevance, the final model was revised to allow this item to load only on the functional factor and not the cognitive factor and, correspondingly, this item was scored only in the HABC-Monitor functional scale score.

In the final model all four factors were significantly correlated except the cognitive and caregiver quality of life factors $(\mathrm{F} 1, \mathrm{~F} 2, \mathrm{r}=0.80 ; \mathrm{F} 1, \mathrm{~F} 3, \mathrm{r}=0.79 ; \mathrm{F} 2, \mathrm{~F} 3, \mathrm{r}=0.77$; $\mathrm{F} 1, \mathrm{~F} 4, \mathrm{r}=0.11$; F2, F4, r = 0.38; and F3, F4, r= 0.38). The fit statistics for the two hypothesized alternative models and the final revised model are shown at the bottom of Table 2. All three models fit the data reasonably well, although the comparative fit index and weighted root mean square residual fit statistics did not quite meet the desired cutoffs. Of note, the only revision we made to the originally hypothesized model 2 was to move the learning gadget item to the functional factor. All items in the final model loaded above 0.40 except two items, ie, item 10 (0.32) and item 25 (0.29). These two items were retained due to their conceptual relevance. Most items loaded above 0.60 . Therefore, the hypothesized four-factor model fit the data reasonably well. The final recommended model contained only one modification to one of the two competing a priori models. The remaining results below apply to the finalized HABC-Monitor scales which were scored by summing the items according to the subscales recommended in our final model.

\section{Reliability and scale score features}

The internal consistency of the HABC-Monitor scales was high (0.73-0.92, Table 3$)$. The observed scale scores covered most of the possible score range, and the mean and standard deviation suggested that the scale scores demonstrated a sufficient dispersion of scores for the purpose of assessing and monitoring the severity of dementia-related symptoms. There were very low (ie, satisfactory) floor and ceiling effects, especially for the scales that the caregiver rated about the patient $(0 \%-18 \%)$. The highest floor effect was for the caregiver quality of life scale, in which $49 \%$ of the caregivers self-reported the lowest (ie, best) possible scores. The three patient symptom scales were moderately correlated $(0.62-0.64)$ as expected, indicating that the domains are related but substantially distinct. Nevertheless, the total score was also highly internally consistent, suggesting that both the HABC-Monitor total scoring and subscale scoring approaches are valid. The caregiver quality of life scale, as expected, demonstrated low correlations with patient symptom scales, in part because caregivers were asked to rate the quality of life items irrespective of whether it was the patient symptoms or other sources of burden that were associated with their quality of life. Therefore, the HABC-Monitor scales demonstrated adequate internal consistency and scale score features including ample dispersion of scores and moderate correlations between patient symptom scales.

\section{Construct validity}

Results in Table 4 showed that at baseline the HABC-Monitor demonstrated construct validity because the highest correlation (0.83) was between the behavioral and psychological subscale of the HABC-Monitor and the NPI total score (0.78), with moderate and significant correlations between the NPI total score and the other two HABC-Monitor patient symptom domain scores (cognitive, 0.62; functional, 0.56). As expected, the HABC-Monitor caregiver quality of life subscale had a significant but relatively lower correlation with the total NPI score (0.35), and a slightly higher correlation with the NPI total score assessment of caregiver distress $(0.44)$. The HABCMonitor caregiver quality of life and the NPI caregiver distress total score are not expected to be highly correlated because the $\mathrm{HABC}-M o n i t o r$ assesses general caregiver quality of life, and does not require the caregiver to identify whether problems with their quality of life are due to patient symptoms or due to other sources. The NPI caregiver distress total score, in contrast, is derived from a more detailed request of the caregiver to assess the impact of 12 patient symptoms on caregiver distress. Like the NPI, the NPI caregiver distress total score demonstrated its highest correlation with the HABC-Monitor behavioral/psychological domain (0.74), and moderate and significant correlations with the HABC-Monitor cognitive 
Table 3 HABC-M scale score features: internal-consistency reliability, score distributions, and inter-score correlations

\begin{tabular}{|c|c|c|c|c|c|c|c|c|c|c|c|c|c|}
\hline \multirow[t]{3}{*}{ HABC-M scales } & \multirow[t]{3}{*}{$\begin{array}{l}\text { Number } \\
\text { of items }\end{array}$} & \multirow{3}{*}{$\begin{array}{l}\text { Reliability } \\
\text { Coefficient } \\
\text { alpha }\end{array}$} & \multicolumn{5}{|c|}{ Score features and distributions } & \multirow[t]{3}{*}{ \% Floor } & \multirow[t]{3}{*}{ \% Ceiling } & \multicolumn{4}{|c|}{$\begin{array}{l}\text { Inter-scale } \\
\text { Spearman r }\end{array}$} \\
\hline & & & \multirow{2}{*}{$\begin{array}{l}\text { Number } \\
\text { possible } \\
\text { levels }\end{array}$} & \multicolumn{4}{|c|}{ Observed score distribution } & & & \multirow[t]{2}{*}{ C } & \multirow[t]{2}{*}{$\mathbf{F}$} & \multirow[t]{2}{*}{ B } & \multirow[t]{2}{*}{$\mathbf{T}$} \\
\hline & & & & Range & Mean & Median & SD & & & & & & \\
\hline \multicolumn{14}{|l|}{ Patient symptoms } \\
\hline Cognitive (C) & 6 & 0.86 & 18 & $0-18$ & 7.4 & 7.0 & 5.4 & 11 & 4 & & & & \\
\hline Functional (F) & 11 & 0.87 & 33 & $0-30$ & 5.9 & 3.0 & 7.0 & 18 & 0 & 0.60 & & & \\
\hline $\begin{array}{l}\text { Behavioral and } \\
\text { psychological (B) }\end{array}$ & 10 & 0.82 & 30 & $0-30$ & 7.3 & 6.0 & 6.3 & 13 & 1 & 0.64 & 0.62 & & \\
\hline Total $(\mathrm{T})$ & 27 & 0.92 & 81 & $0-75$ & 20.6 & 17.0 & 16.3 & 3 & 0 & 0.88 & 0.82 & 0.88 & \\
\hline $\begin{array}{l}\text { Caregiver quality } \\
\text { of life }(\mathrm{Q})\end{array}$ & 4 & 0.73 & 12 & $0-11$ & 1.8 & 1.0 & 2.5 & 49 & 0 & 0.13 & 0.33 & 0.33 & 0.29 \\
\hline
\end{tabular}

Notes: \% Floor is the percentage of caregivers who reported the lowest (best) possible score. \% Ceiling is the percentage of caregivers who reported the highest (worst) possible score.

Abbreviation: HABC-M, Healthy Aging Brain Care Monitor.

and functional domain scores. Therefore, the HABC-Monitor demonstrated good construct validity.

\section{Sensitivity to change}

The sensitivity to change results are shown in Table 5. The first two rows of data display descriptive statistics that help to describe the "reliable change" groups. Specifically, for the group that declined in NPI total score by more than 1.0 SEM from baseline to 3 months, their mean NPI total score was 11.3 at baseline (time 1) and became worse (20.9) 3 months later at time 2 . The NPI stable group retained low (relatively good) mean NPI scores from time 1 to time 2 (4.9 and 5.1, respectively). In contrast, the NPI improved group started at a worse level than the other two groups at baseline (29.8) but improved to a mean score of 18.6 when assessed 3 months later. Because the NPI total score is a gold standard for measuring the behavioral and psychological symptoms related to dementia through the caregiver, sensitivity-to-change validity for the HABC-Monitor would be indicated by significant differences between the NPI reliable change groups on

Table 4 Construct validity of HABC-Monitor

\begin{tabular}{lll}
\hline HABC-Monitor scales & $\begin{array}{l}\text { NPI } \\
(\mathbf{n}=17 \mathbf{I})\end{array}$ & $\begin{array}{l}\text { NPI-cg } \\
(\mathbf{n}=\mathbf{I 7 I})\end{array}$ \\
\hline Patient symptoms (reported by caregiver) & & \\
$\quad$ Cognitive & $0.62^{* * *}$ & $0.5 I^{* * *}$ \\
Functional & $0.56^{* * *}$ & $0.53^{* * *}$ \\
Behavioral/psychological & $0.83^{* * *}$ & $0.75^{* * *}$ \\
$\quad$ Total score & $0.78^{* * *}$ & $0.69^{* * *}$ \\
Caregiver quality of life (reported by caregiver) & $0.35^{* * *}$ & $0.44^{* * *}$ \\
\hline
\end{tabular}

Notes: Values represent Spearman correlation coefficients. NPI = Neuropsychiatric Inventory (NPI) total score, which is a gold standard of caregiver assessment of the patient's frequency and severity of behavioral/psychological symptoms relevant to dementia. NPI-cg = NPI caregiver distress total score, an indicator of caregiver assessment of caregiver distress due to the patient's symptoms. ${ }^{* * * P} P<0.001$. Abbreviation: $\mathrm{HABC}$, Healthy Aging Brain Care. the mean change scores of the HABC-Monitor scales. The analysis of variance omnibus $P$ value in Table 5 shows that the three NPI groups demonstrate significantly different mean change scores for each and every HABC-Monitor scale score except for the HABC-Monitor caregiver quality of life scale. As expected, of the three HABC-Monitor patient symptom subscales, the behavioral and psychological subscale displayed the strongest differences, including significant Tukey-Kramer post hoc pairwise differences among all three NPI reliable change groups. The HABC-Monitor cognitive subscale significantly separated the improved group from the declined and stable groups but did not separate the declined group from the stable group. The HABC-Monitor functional subscale significantly separated only the improved group from the declined group, and showed marginal ability $(P=0.058)$ to distinguish the declined group from the stable group. The direction and magnitude of HABC-Monitor change score effect sizes were consistent with theory. For example, the largest effect sizes for the change scores were for HABC-Monitor behavioral/psychological change scores, which demonstrated moderate improvement in the NPI improved group (0.53) and moderate decline in the NPI declined group $(-0.43)$. The HABC-Monitor caregiver quality of life score showed slight to moderate declines, according to effect size, in all three NPI groups. It is not clear why this was, except it should be noted that the mean HABC-Monitor caregiver quality of life score at time 2 was similar for the declined and improved groups (mean $=2.6$ for both groups). Furthermore, the quality of life score was indeed lower (ie, better) at baseline for the stable group (1.0) and improved group (1.7) compared with the declined group (2.6). Caregiver quality of life seems to have gotten substantially worse over time (effect size $=-0.70$ ) for the NPI stable group, perhaps because the caregivers had a 


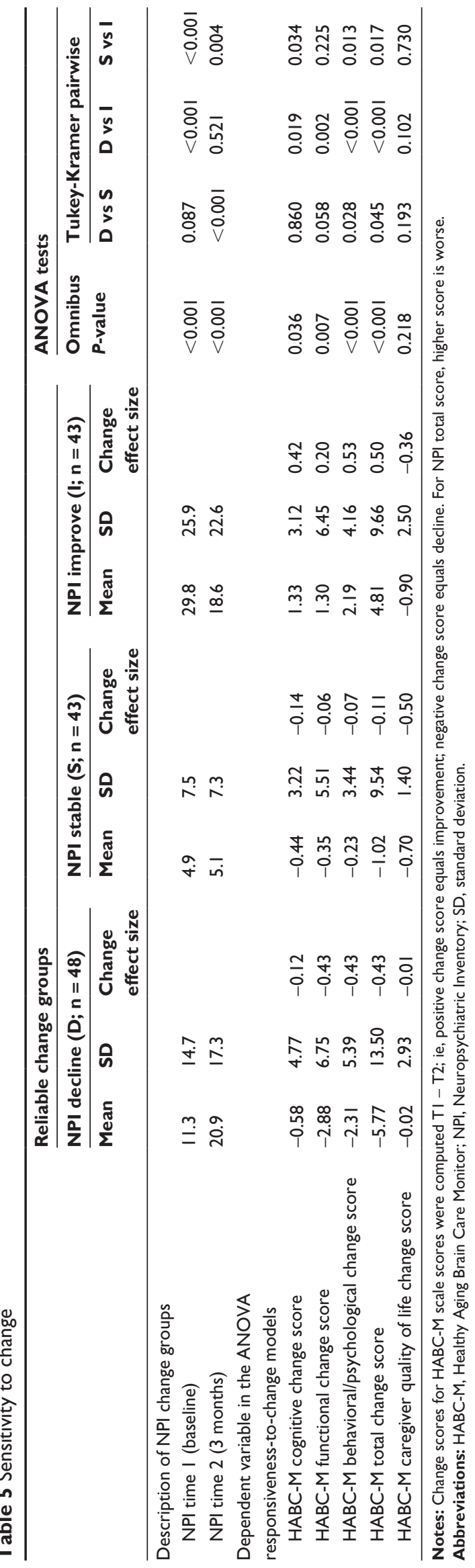

chance to focus on themselves while the patient was stable; nevertheless, the effect sizes for the three groups should not be overly interpreted because the NPI change groups did not differ significantly on the mean caregiver quality of life change scores as indicated by the $P$ values (Table 5). In summary, the HABC-Monitor showed good sensitivity to change indicated by significant omnibus and pairwise differences between the NPI reliable change groups on the HABC-M patient symptom scales.

\section{Known-groups validity}

The extent to which the HABC-Monitor scales separate the known groups of cognitive impairment, defined by MMSE cognitive-impairment severity score groups, is evidence of known-groups validity. Separation of consensus clinicianbased diagnoses with HABC-Monitor scores is also evidence of known-groups validity. Table 6 shows that the omnibus test was significant for all of the HABC-Monitor patient symptom scales with respect to separating the MMSE groups. All of the six pairwise differences between MMSE groups were significantly different on at least one of the three HABC-Monitor patient subscale scores. As expected, the HABC-Monitor cognitive scale was the strongest scale for separating the MMSE groups, and it alone separated the normal and mild MMSE groups. Despite a small sample size for the MMSE severe group ( $n=10)$, the severe MMSE group was significantly different from the normal and mild MMSE groups on all HABC-Monitor patient scales and was significantly different from the moderate MMSE group on the HABC-Monitor functional and total scores but not the HABC-Monitor cognitive score, indicating the usefulness of having distinct subscales on the HABC-Monitor.

The clinical diagnostic groups were significantly different on all HABC-Monitor patient symptom scales with respect to the omnibus test. All pairs of the diagnostic groups were significantly different on the mean HABC-Monitor cognitive score. The HABC-Monitor functional, behavioral/psychological, and total scores were able to distinguish the dementia diagnostic group from the normal and mild cognitive impairment diagnostic groups clearly, but could not distinguish the normal diagnostic group from the mild cognitive impairment diagnostic group.

Based on the eta-squared values, MMSE group membership was most highly associated with the HABC-Monitor cognitive and functional domains. The clinical diagnostic groups were more highly associated with the HABC-Monitor cognitive domain than the functional or behavioral and psychological domains. In summary, the HABC-Monitor showed good known-groups validity, indicated by significant 


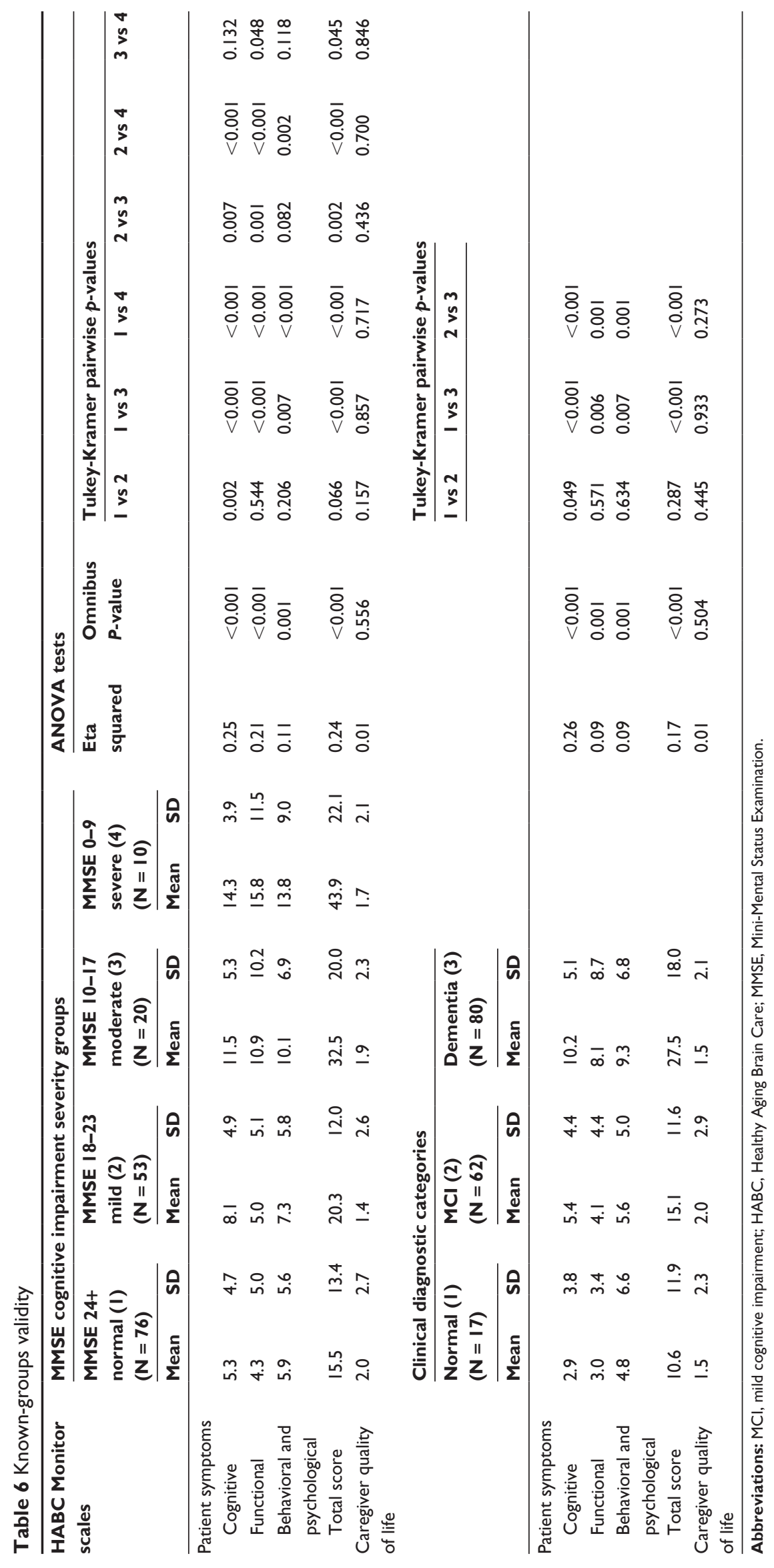


omnibus and pairwise differences between MMSE and clinician-based diagnostic groups.

\section{Operating characteristics}

For predicting dementia (versus mild cognitive impairment or normal), the AUROC (and 95\% confidence interval) for the 10-item HABC-Monitor behavioral and psychological scale $(0.67 ; 0.59-0.75)$ was comparable with the AUROC for the more extensive NPI total score $(0.66 ; 0.58-0.74)$. The HABCMonitor total score was slightly better but comparable $(0.72$; 0.65-0.80). The total score for the 30-item MMSE had an AUROC of $0.88(0.83-0.93)$ while the six-item HABC-Monitor cognitive scale demonstrated an AUROC of 0.77 (0.70-0.84)

For predicting normal (versus mild cognitive impairment or dementia), the AUROC for the 10-item HABC-Monitor behavioral and psychological scale was similar $(0.67 ; 0.52-0.82)$ to that of the NPI total score $(0.63 ; 0.47-0.81)$, and the HABC-Monitor total score was only slightly better $(0.73 ; 0.59-0.86)$ than the HABC-Monitor behavioral and psychological scale. The AUROC was 0.91 (0.84-0.97) and 0.79 (0.68-0.90), respectively, for the MMSE total and the HABC-Monitor cognitive score.

Screening studies often report the findings for separating extreme groups (dementia and normal) because the results show, perhaps inappropriately, inflated operating characteristics compared with when the mild cognitive impairment group is included, as would be the case in usual practice. For purposes of comparison with published studies that compare dementia versus normal groups, the HABC-Monitor cognitive score showed an AUROC of $0.88(0.78-0.98)$ and the MMSE was 0.97 (0.94-1.00). In summary, this preliminary report of the operating characteristics of the HABC-Monitor suggests good performance compared with the lengthier NPI. Performance was encouraging and adequate compared with the MMSE considering that the MMSE and clinical diagnoses were administered at the same time, which was 1-12 months earlier than the collection of HABC-Monitor data.

\section{Sensitivity analyses}

We thank a reviewer for suggesting that we examine possible effects of race and education on results. We re-estimated Cronbach's coefficient alpha and construct validity in three different subgroup dichotomizations, ie, white versus nonwhite patients, white versus nonwhite caregivers, and caregivers with less than 12 years of education versus higher education. (We did not collect patient education level). The results, ie, effect sizes and hypothesis-testing conclusions, did not differ by subgroups. For example, Cronbach's coefficient alpha differed very little by subgroups, and all subgroup alphas continued to be in the range of $0.73-0.92$, as they were in the total sample.

We thank another reviewer for prompting us to address whether the NPI "improved" group was due to intervention effects or due to severely impaired individuals becoming so apathetic that their previous behavioral and psychological symptoms were lessened. As described later in this paper, we believe that the improved group is a reflection of the effect of the collaborative care models for dementia and depression delivered by the selected memory care practice for this study. We performed an additional analysis, the results of which ruled out the interesting apathy hypothesis. The HABC-Monitor apathy item (ie, item 18, the anhedonia item) improved from time 1 to time 2 in the NPI improved group (item mean decreased from 1.8 to 1.4 ) and worsened in the NPI declined group (item mean increased from 1.0 to 1.5).

\section{Discussion}

The HABC-Monitor demonstrates a high degree of reliability and validity (including good sensitivity to change) for assessment and monitoring of the severity of dementiarelated symptoms through the input of informal caregivers. The instrument does this while maintaining the brevity and simple format necessary for use in clinical practice. Thus, the tool appears to exhibit both research validity and has the potential to be used as a simple and practical "blood pressure cuff".

The HABC-Monitor exhibits good data quality, including adequate item and scale score variability and low missing data rates. The hypothesized four-factor solution for the HABC-Monitor items demonstrates reasonably good fit for both of the two a priori alternative models. The final recommended model underwent only one minor revision, namely, the learning tools item was moved to the functional factor in the a priori model that placed the anhedonia item in the behavioral and psychological factor. Perhaps future revisions of the HABC-Monitor by our team or other research/clinical groups will improve the fit of the factor model even further, so that all major fit indices show good fit while maintaining brevity to maximize use of the instrument in routine clinical practice.

The HABC-Monitor demonstrates good sensitivity to change and known-groups validity. For construct validity, the 10-item behavioral and psychological scale of the HABC-Monitor was highly correlated (0.83) with the NPI total score, despite the fact that the NPI is a more extensive questionnaire that accounts for both frequency and severity, whereas the HABC-Monitor tool accounts only for the 
frequency of problems. The 31-item HABC-Monitor also met our initial criterion of brevity, taking on average 6 minutes to complete.

Although not designed to be a screening study, the AUROC findings provide preliminary evidence that the HABC-Monitor, especially the cognitive scale, may be useful for the purposes of screening, despite the fact that the HABC-Monitor was developed primarily for the purpose of assessing and monitoring the severity of dementia-related symptoms. This may be largely due to the fact that the cognitive scale contains six of the eight items of the AD8, a dementia screening tool that has been validated in clinical and research samples against gold standard clinical and neuropsychological evaluations ${ }^{12,13}$ and against biomarkers of Alzheimer's disease. ${ }^{26}$ The operating characteristics of the HABC-Monitor, when compared with the NPI and MMSE, were encouraging, given that the gold standard clinical diagnoses were not obtained as part of this study design. Instead, the gold standard diagnoses were obtained, along with the MMSE, from the HABC intake and Consortium to Establish a Registry for Alzheimer's disease data 1-12 months earlier than the present study's baseline instruments (HABC-Monitor, NPI). For the data that were administered at the same time as the HABC-Monitor (ie, NPI), the HABC-Monitor behavioral/psychological and total scores demonstrated AUROC values that were comparable with AUROC values of the more detailed, but more time-consuming, NPI which is based on structured interview. The NPI is highly reliable and valid, and extremely useful for research studies and perhaps for clinic settings that have protected or funded time for longer instruments. The NPI-Q ${ }^{15}$ is a briefer and clinically practical version of the NPI; however, for the purpose of monitoring symptoms with a brief tool that includes not only behavioral and psychological but also cognitive and functional domains, the HABC-Monitor demonstrates validity for research while also being useful for monitoring symptoms in the clinic.

The closest existing multidimensional instrument we found to the HABC-Monitor for capturing dementia-related symptoms through the caregiver in a brief tool is the Dementia Severity Scale. ${ }^{11}$ This 47 -item scale measures symptoms in the cognitive, functional, and behavioral domains, and has demonstrated good internal consistency, test-retest reliability, and construct validity. ${ }^{11}$ The Dementia Severity Scale is slightly longer than the HABC-Monitor (47 versus 31 items). An important content difference between the HABC-Monitor and the Dementia Severity Scale is that the latter does not contain any items that assess mood (depressive or anxiety) symptoms, which are common in patients with cognitive impairment. The Relevant Outcome Scale for Alzheimer's Disease (ROSA) is a 16-item observer rating scale but requires a trained observer. ${ }^{27}$ The ROSA uses one to five items to tap symptom domains of patient cognition, communication, function/activities of daily living, behavior, quality of life, and caregiver burden. ${ }^{27}$ The ROSA demonstrated good internal consistency and inter-rater reliability and validity, including sensitivity to change. However, the ROSA demonstrated only two factors in a factor analysis, one factor related to cognition, communication, function, quality of life, and caregiver burden, and another factor consisting of all behavior items. In addition, the ROSA took an average of 13-15 minutes to complete. Furthermore, important from our perspective, like the Dementia Severity Scale, the ROSA does not include depression or anxiety symptoms in the psychological or behavioral domain.

The HABC-Monitor is brief enough to be printed on one side of a single page in order to maximize clinical utility, yet it includes enough items to demonstrate confirmed multidimensionality in a factor analysis in which each domain exhibits its own factor.

\section{Limitations}

The MMSE data and clinical diagnoses were collected up to one year earlier than the baseline administration of the HABC-Monitor. Therefore, our results for known-groups validity and operating characteristics of the HABC-Monitor may have underestimated true magnitudes. Although the study design was severely biased against the HABC-Monitor for these two pieces of validity, the findings were positive for the HABC-Monitor. The known-groups validity was strong. The preliminary results for operating characteristics were encouraging because the HABC-Monitor performance was comparable with the AUROC when administered at the same time as the lengthier gold standard instrument for behavioral and psychological symptoms (NPI). Although the AUROC values were somewhat higher for the MMSE than for the HABC-M cognitive scale, this was expected because the MMSE was administered during the same visit as that during which the clinical diagnoses were made. The absolute values of the AUROC for the HABC-Monitor cognitive scale were reasonably high given the circumstance that the clinical diagnoses were rendered up to one year earlier than administration of the HABC-Monitor. Furthermore, the six-item HABC-M cognitive scale is much briefer than the 30-item MMSE. Therefore, the performance of the HABC-M cognitive scale compared with the MMSE is 
encouraging; however, these results are only preliminary and more validation is needed. Future studies should assess the diagnostic accuracy of the HABC-Monitor compared with other instruments, such as the MMSE, as part of a more suitable screening study design in which ample patients are represented for each of three groups (normal, mild cognitive impairment, and dementia), and in which all instruments are assessed at the same time as the gold standard clinical diagnoses.

The development of the HABC-Monitor was in a memory care practice setting where the prevalence of dementia and other cognitive impairment is high. Validating the HABCMonitor in other settings such as primary care is a reasonable next step. The sensitivity to change of the HABC-Monitor was evaluated over a 3-month period. Future projects will validate the $\mathrm{HABC}$-Monitor in primary care to assess sensitivity to change over longer periods. Thus, although the HABC-Monitor demonstrated good reliability and validity in the present study, more validation studies are needed.

\section{Clinical implications}

Patients and informal caregivers enrolled in this study were recruited from our Healthy Aging Brain Center. There were possible effects of the memory care practice on the quality of lives of not only the patients but also the informal caregivers who were enrolled in the study. The biopsychosocial interventions that are delivered by our memory care practice are delivered to both the patients and their informal caregivers. Such an intervention is based on our previous successful demonstration of the collaborative care model for dementia. ${ }^{4,6}$ This center is a memory care practice that has translated the collaborative care models for dementia ${ }^{4}$ and depression ${ }^{28}$ into a self-sustained clinical program. ${ }^{6}$

The collaborative care models for dementia and depression have been successfully able to improve the behavioral, psychological, and mood symptoms of patients suffering from dementia or depression. Based on the present findings and the published literature on the dementia collaborative care model, ${ }^{4}$ as well as our clinical experience in the Healthy Aging Brain Center, ${ }^{6}$ we believe that the HABCMonitor will benefit patients with Alzheimer's disease, their informal caregivers, and dementia care clinicians of all disciplines by providing clinicians with more frequent, more reliable, and more useful information, making it possible to modify care plans as needed to target problem symptoms more effectively. Better dementia symptom management improves quality of life for both the patient and their informal caregiver.

\section{Disclosure}

Author (MB) was the principal investigator of this study. This work was funded by grants from National Institute on Aging (P30AG024967), National Institute of Mental Health (R24MH080827), and Agency for Healthcare Research and Quality (R01 HS019818-01). Dr Galvin's time contribution was supported as part of a grant from National Institute on Aging (R01 AG040211). The HABC-Monitor is a copyrighted instrument by Drs Boustani, Galvin and Callahan and the Indiana University School of Medicine. The HABCMonitor and scoring rules are available at http://www. wishard.edu/our-services/senior-care/healthy-aging-braincenter/cgm

\section{References}

1. Boustani M, Peterson B, Hanson L, Harris R, Lohr KN. Screening for dementia in primary care: A summary of the evidence for the US Preventive Services Task Force. Ann Intern Med. 2003;138(11):927-937.

2. Holsinger T, Deveau J, Boustani M, Williams JW Jr. Does this patient have dementia? JAMA. 2007;297(21):2391-2404.

3. Hinton L, Franz C, Reddy G, Flores Y, Kravitz R, Barker J. Practice constraints, behavioral problems, and dementia care: primary care physicians' perspectives. J Gen Intern Med. 2007;22(11):1487-1492.

4. Callahan CM, Boustani MA, Unverzagt FW, et al. Effectiveness of collaborative care for older adults with Alzheimer disease in primary care. JAMA. 2006;295(18):2148-2157.

5. Vickrey BG, Mittman BS, Connor KI, et al. The effect of a disease management intervention on quality and outcomes of dementia care. Ann Intern Med. 2006;145(10):713-726.

6. Boustani MA, Sachs GA, Alder CA, et al. Implementing innovative models of dementia care: the Healthy Aging Brain Center. Aging Ment Health. 2011;15(1):13-22.

7. Callahan CM, Boustani MA, Weiner M, et al. Implementing dementia care models in primary care settings: the aging brain care medical home. Aging Ment Health. 2011;15(1):5-12.

8. Morris JC. The Clinical Dementia Rating (CDR): current version and scoring rules. Neurology. 1993;43(11):2412-2414.

9. Cummings JL, Mega M, Gray K, Rosenberg-Thompson S, Carusi DA, Gornbein J. The Neuropsychiatric Inventory: comprehensive assessment of psychopathology in dementia. Neurology. 1994;44(12): 2308-2314.

10. Clark CM, Ewbank DC. Performance of the dementia severity rating scale: a caregiver questionnaire for rating severity in Alzheimer disease. Alzheimer Dis Assoc Disord. 1996;10(1):31-39.

11. Harvey PD, Moriarty PJ, Kleinman L, et al. The validation of a caregiver assessment of dementia: the Dementia Severity Scale. Alzheimer Dis Assoc Disord. 2005;19(4):186-194.

12. Galvin JE, Roe C, Coats M, et al. The AD8: a brief informant interview to detect dementia. Neurology. 2005;65(4):559-564.

13. Galvin JE, Roe CM, Xiong C, Morris JC. Validity and reliability of the AD8 informant interview in dementia. Neurology. 2006;67(11): 1942-1948.

14. Spitzer RL, Kroenke K, Williams JBW. Validation and utility of a selfreport version of PRIME-MD: the PHQ primary care study. JAMA. 1999;282(18):1737-1744.

15. Kaufer DI, Cummings JL, Ketchel P, et al. Validation of the NPI-Q, a brief clinical form of the Neuropsychiatric Inventory. JNeuropsychiatry Clin Neurosci. 2000;12(2):233-239.

16. Min LC, Wenger NS, Reuben DB, Saliba D. A short functional survey is responsive to changes in functional status in vulnerable older people. J Am Geriatr Soc. 2008;56(10):1932-1936. 
17. Folstein MF, Folstein SE, McHugh PR. Mini-mental state: a practical method for grading the cognitive state of patients for the clinician. Psychiatr Res. 1975;12(3):189-198.

18. Muthen LK, Muthen BO. MPLUS User's Guide. 5th ed. Los Angeles, CA: Muthen and Muthen; 1998-2007.

19. Hu LT, Bentler PM. Cutoff criteria for fit indexes in covariance structure analysis: conventional criteria versus new alternatives. Structural Equation Modeling. 1999;6(1):1-55.

20. Yu CY. Evaluating cutoff criteria of model fit indices for latent variable models with binary and continuous outcomes. Doctoral Dissertation, University of California, Los Angeles, CA, 2002. Available from: http:// www.statmodel.com/download/Yudissertation.pdf. Accessed April 19, 2012.

21. Cronbach LJ. Coefficient alpha and the internal structure of tests. Psychometrika. 1951;16(3):297-334.

22. Nunnally JC, Bernstein IH. Psychometric Theory. 3rd ed. New York, NY: McGraw-Hill; 1994.

23. Guyatt G, Walter S, Norman G. Measuring change over time: assessing the usefulness of evaluative instruments. J Chronic Dis. 1987;40(2): 171-178.
24. Deyo RA, Diehr P, Patrick DL. Reproducibility and responsiveness of health status measures. Statistics and strategies for evaluation. Control Clin Trials. 1991;12(4 Suppl):142S-158S.

25. Cummings JL. The Neuropsychiatric Inventory: assessing psychopathology in dementia patients. Neurology. 1997;48(5 Suppl 6): S10-S16.

26. Galvin JE, Fagan AM, Holtzman DM, Mintun MA, Morris JC. Relationship of dementia screening tests with biomarkers of Alzheimer's disease. Brain. 2010;133(11):3290-3300.

27. Holthoff VA, Ferris S, Ihl R, et al. Validation of the relevant outcome scale for Alzheimer's disease: a novel multidomain assessment for daily medical practice. Alzheimers Res Ther. 2011;3(5):27.

28. Unützer J, Katon W, Callahan CM, et al. Collaborative care management of late-life depression in the primary care setting. JAMA. 2002;288(22):2836-2845. 


\section{Appendix}

Items of the HABC-Monitor

Over the past two weeks, how often did your loved one have

problems with:

\section{Cognitive items}

I Judgment or decision-making

2 Repeating the same things over and over, such as questions or stories

3 Forgetting the correct month or year

4 Handling complicated financial affairs, such as balancing checkbook, income taxes, and paying bills

5 Remembering appointments

6 Thinking or memory

Functional items

7 Learning how to use a tool, appliance, or gadget

8 Planning, preparing, or serving meals

9 Taking medications in the right dose at the right time

10 Walking or physical ambulation

II Bathing

12 Shopping for personal items like groceries

13 Housework or household chores

14 Leaving her/him alone

$15 \mathrm{Her} /$ his safety

$16 \mathrm{Her} /$ his quality of life

17 Falling or tripping

Behavioral and psychological items

18 Less interest or pleasure in doing things, hobbies, or activities

19 Feeling down, depressed, or hopeless

20 Being stubborn, agitated, aggressive, or resistive to help from others

2I Feeling anxious, nervous, tense, fearful or panic

22 Believing others are stealing from them or planning to harm them

23 Hearing voices, seeing things, or talking to people who are not there

24 Poor appetite or overeating

25 Falling asleep, staying asleep, or sleeping too much

26 Acting impulsively, without thinking through the consequences of her/his actions

27 Wandering, pacing, or doing things repeatedly

Caregiver quality of life items

Over the past two weeks, how often did you have problems with:

28 Your quality of life

29 Your financial future

30 Your mental health

3I Your physical health

Note: A full copy of the HABC-Monitor is available at http://www.wishard.edu/ our-services/senior-care/healthy-aging-brain-center/cgm.

\section{Publish your work in this journal}

Clinical Interventions in Aging is an international, peer-reviewed journal focusing on evidence-based reports on the value or lack thereof of treatments intended to prevent or delay the onset of maladaptive correlates of aging in human beings. This journal is indexed on PubMed Central, MedLine, the American Chemical Society's 'Chemical Abstracts Ser-

\section{Dovepress}

vice' (CAS), Scopus and the Elsevier Bibliographic databases. The manuscript management system is completely online and includes a very quick and fair peer-review system, which is all easy to use. Visit http://www.dovepress.com/testimonials.php to read real quotes from published authors. 